\title{
Prevalencia aumentada de enfermedad celiaca en familiares de primer y segundo grado: descripción de una familia con 19 miembros estudiados
}

\author{
L. Rodrigo, D. Fuentes, S. Riestra, P. Niño, N. Álvarez, A. López-Vázquez y y C. López-Larrea ${ }^{1}$ \\ Servicios de Digestivo e Inmunología. Hospital Universitario Central de Asturias. Oviedo
}

\section{RESUMEN}

Introducción: la enfermedad celiaca (EC) es un proceso autoinmune, desencadenado por la ingesta del gluten contenido en la mayor parte de los cereales, que afecta a individuos genéticamente predispuestos. Por todo ello, muestra una clara tendencia familiar, centrada fundamentalmente en marcadores del sistema HLA de clase II.

Objetivos: nos propusimos en el presente trabajo analizar la prevalencia de EC en una familia extensa, a partir de un caso índice fallecido hacía unos años, como consecuencia de padecer la misma enfermedad, complicada además con el desarrollo de un tumor maligno del intestino delgado, del tipo del adenocarcinoma.

Métodos: se estudiaron un total de 19 miembros. Se les realizó un protocolo diagnóstico que incluía un historia clínica detallada, junto con una hemograma y estudio de coagulación, una bioquímica amplia incluyendo pruebas de función hepática, estudio sérico del metabolismo del hierro, niveles circulantes de ácido fólico y vitamina $\mathrm{B}_{12}$, pruebas de función tiroidea, determinación de la transglutaminasa tisular y marcadores genéticos (DQ2 y DQ8). En los casos sospechosos y para su confirmación se realizó gastroscopia completada con toma de biopsias duodenales múltiples.

Resultados: encontramos una prevalencia global de EC en 9/19 de los familiares estudiados, lo que representa un $47,4 \%$, distribuidos de la siguiente manera en función del parentesco con el caso índice: cuatro de siete hermanos (57\%); uno de tres hijos (33,3\%); tres de ocho sobrinos $(37,5 \%)$; y el único sobrino-nieto estudiado de nueve años de edad, también estaba afecto.

Conclusiones: de todo ello se deduce la necesidad de hacer estudios amplios familiares, cada vez que se diagnostica un paciente de enfermedad celiaca, incluyendo familiares de primero y segundo grado, dada la relativa facilidad actual para llevarlos a cabo y la elevada prevalencia encontrada.

Palabras clave: Enfermedad celiaca. Estudio familiar. Despistaje clínico-serológico. Familiares de $1^{\text {er }}$ y $2^{\circ}$ grado.

Recibido: 27-09-06.

Aceptado: 01-12-06.

Correspondencia: Luis Rodrigo. Servicio de Digestivo. Hospital Universitario Central de Asturias. C/ Celestino Villamil, s/n. 33006 Oviedo. Fax: 985-27-36-14. e-mail: 1rodrigos@terra.es

\begin{abstract}
Introduction: celiac disease $(\mathrm{CD})$ is an autoimmune condition that is triggered by the ingestion of gluten, a substance present in most cereals, and that affects genetically predisposed individuals. As a result, this condition is clearly familial, and mainly associated with HLA class II markers.

Objectives: in this work we set out to analyze the prevalence of $\mathrm{CD}$ in an extensive family based on an index subject who had already died from this disease a few years ago, where $C D$ had been complicated by the development of a small-bowel malignancy, namely an adenocarcinoma.

Methods: nineteen members were studied. They all were subjected to a diagnostic protocol including a detailed medical history, hemogram, coagulation tests, and blood biochemistry (including liver function tests, serum iron metabolism, circulating folic acid and vitamin $B_{12}$ levels, thyroid function tests, tissue transglutaminase measurement, and genetic markers (DQ2 and DQ8). Suspect cases underwent gastroscopy plus multiple duodenal biopsy for confirmation.

Results: overall we encountered CD in 9/19 studied members, which represents $47.4 \%$ with the following distribution according to degree of kinship -four of seven siblings (57\%); one of three children (33.3\%); three of eight nephews and nieces (37.5\%), and the only grandnephew, who was 9 years old.

Conclusions: from all this it may be seen that family studies are needed every time a patient is diagnosed with celiac disease; these studies should include both first- and second-degree relatives, given the high prevalence encountered and the fact that these tests are relatively straighforward to perform.
\end{abstract}

Key words: Celiac disease. Family study. Clinical-serological screening. First- and second-degree relatives.

Rodrigo L, Fuentes D, Riestra S, Niño P, Álvarez N, LópezVázquez A, López-Larrea C. Prevalencia aumentada de enfermedad celiaca en familiares de primer grado: descripción de una familia con 19 miembros estudiados. Rev Esp Enferm Dig 2007; 99: 149-155. 


\section{INTRODUCCIÓN}

La enfermedad celiaca (EC) es un proceso de base autoinmune que afecta primariamente al tubo digestivo, con especial afectación del intestino delgado, caracterizada por la presencia de inflamación crónica de la mucosa duodenal, que se asocia frecuentemente, aunque no siempre, con la presencia de atrofia de las vellosidades intestinales, cursando en ocasiones con un cuadro de malabsorción, junto con una serie de manifestaciones clínicas diversas, que pueden aparecer tanto en la infancia, como a cualquier edad de la vida. Actualmente se la conoce como una enfermedad frecuente, pero sigue siendo muy desconocida por parte de muchos médicos y claramente infradiagnosticada en el adulto (1-3).

Se desencadena por la ingestión del gluten y otras proteínas relacionadas presentes en el trigo, centeno y cebada, exclusivamente. Afecta a individuos genéticamente predispuestos, portadores del antígeno DQ2, expresado en el 95\% de los pacientes y el resto son DQ8 (+). La eliminación de la dieta de los alimentos que contienen gluten conduce a una mejoría clínica a histológica permanente $(4,5)$.

Existe una fuerte susceptibilidad genética en el desarrollo de EC, como lo confirma la elevada concordancia observada en gemelos monozigóticos, que llega hasta un 75\% (6).

Se encuentra una elevada prevalencia de EC, en familiares de primer y segundo grado que oscila del 4-12\%, como describimos previamente (7). Esta relación es debi$\mathrm{da}$, en gran parte, a la existencia de una importante base genética condicionada por la presencia de los antígenos de clase HLA-II anteriormente citados (DQ2 y DQ8).

Estudios previos han confirmado la existencia de un riesgo aumentado de aparición de diversos tumores digestivos y extradigestivos asociados a la EC, que aparecen generalmente en pacientes diagnosticados en la edad adulta. Sin embargo, la presencia del linfoma intestinal debe seguir siendo considerada como una rara complicación de esta enfermedad $(8,9)$.

\section{PACIENTES Y MÉTODOS}

Se realizó un estudio familiar de enfermedad celiaca que se llevó a cabo de forma ambulatoria, en una consulta monográfica de EC, del Servicio de Digestivo del Hospital Central de Asturias en Oviedo, invitando a participar a todos los familiares de primer grado a partir de un caso índice fallecido hace 15 años por una enfermedad celiaca, con un tumor maligno de intestino delgado asociado.

Participaron en el estudio voluntariamente, previa citación, un total de 19 miembros distribuidos de la siguiente manera: seis hermanos, tres hijos, ocho sobrinos y un sobrino-nieto.

A todos los familiares estudiados se les realizó una historia clínica detallada, junto con una exploración física completa y una serie de pruebas analíticas entre las que se incluían las siguientes: hemograma completo y estudio de coagulación, bioquímica amplia incluyendo pruebas de función hepática, estudios de función tiroidea, estudios analíticos del metabolismo del hierro (sideremia, ferritina e índice de saturación de transferrina), así como determinación de los niveles séricos de ácido fólico y vitamina- $\mathrm{B}_{12}$.

Para la serología de EC se determinaron única y exclusivamente los anticuerpos anti-transglutaminasa tisular tipo 2, de clase IgA (TTG), mediante una técnica de ELISA comercial (Orgentec, Diagnostika GMBH. Mainz, Alemania, que emplea una TTG humana recombinante, extraída de una línea celular embrinaria de riñón, 293-EBNA). Los sueros fueron diluidos a diversas concentraciones con solución de Tween 20 (TET), e incubadas durante 1,5 horas con TTG humana, a temperatura ambiente. Las absorbancias de color obtenidas se leyeron en colorímetro, a una longitud de onda de $450 \mathrm{~nm}$. Se consideraron valores positivos, cuando eran mayores de $1 \mathrm{U} / \mathrm{ml}$ (10).

Para llevar a cabo el estudio de los marcadores de susceptibilidad genética, se extrajeron $2 \mathrm{ml}$ de sangre periférica que se mezclaron con $2 \mathrm{ml}$ de alcohol absoluto y se almacenaron hasta que las muestras fueron analizadas. Para la realización de la prueba, se eliminó el alcohol lavando por dos veces los extractos con solución de PBS (buffer fosfato) y las muestras obtenidas fueron analizadas para la determinación del HLA-DQ2 por técnica de PCR-SSP (secuencia específica de cebadores) y cuando estos fueron negativos, se determinaron los DQ8 por el mismo procedimiento, de acuerdo con un método previamente descrito (11).

En los casos con sospecha clínico-analítica fundada de enfermedad celiaca se realizó una endoscopia digestiva alta con toma de biopsias múltiples duodenales (4 como mínimo) mediante un fibroduodenoscopio marca Fujinon, modelo EG-250WR. El estudio morfológico de las biopsias se llevó a cabo en cortes de parafina teñidos con los colorantes habituales de hematoxilina/eosina. El recuento de linfocitos intraepiteliales se realizó con técnicas especiales de inmunohistoquímica para CD-8 y se midió su presencia, considerándolos positivos cuando su densidad era mayor o igual de 40 por cada 100 enterocitos. El grado de atrofia de la mucosa (parcial, subtotal, total) se expresó al igual que otros hallazgos histológicos de EC, tales como la hiperplasia de criptas y la presencia de infiltrado inflamatorio a nivel de la lámina propia, mediante la clasificación de Marsh de 1990 (12).

Fueron diagnosticados como pacientes con EC todos aquellos familiares que presentaban clínica y analítica compatibles, junto con marcadores genéticos positivos, TTG aumentada y lesiones histológicas compatibles.

\section{RESULTADOS}

De los 19 familiares estudiados, hemos encontrado un total de 9 pacientes afectos de enfermedad celiaca, lo que representa una prevalencia muy elevada, del orden del 
$47,4 \%$, distribuidos de la siguiente manera: $4 / 7$ hermanos $(57 \%)$; $1 / 3$ hijos $(33,3 \%) ; 3 / 8$ sobrinos $(37,5 \%)$ y un sobrino-nieto, que fue el único estudiado.

El árbol genealógico de los familiares estudiados, señalando los individuos sanos y familiares afectos de EC, se representan gráficamente. Los padres y dos hermanos del caso índice no pudieron ser analizados, pues habían fallecido varios años antes de llevar a cabo el presente estudio (Fig. 1).

Las características, clínicas, genéticas, serológicas e histológicas de los familiares afectos se agrupan de forma comparativa y detallada (Tabla I).

La descripción detallada del caso índice y familiares afectos, se describen a continuación:

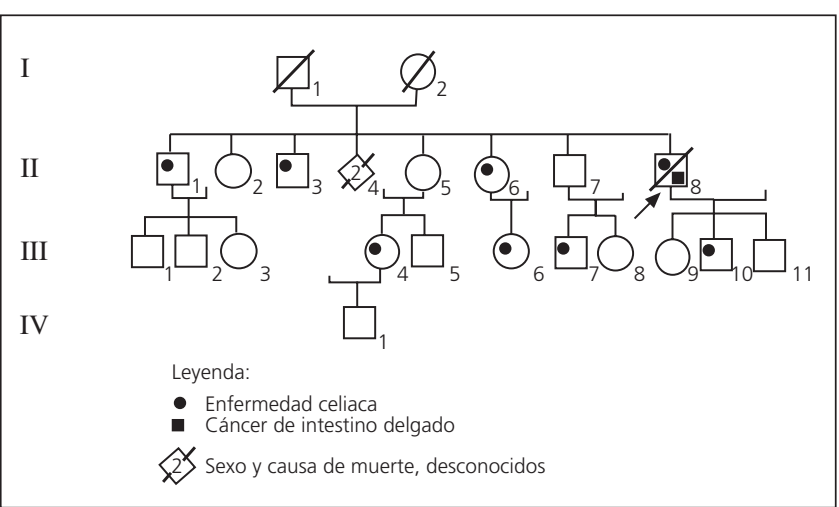

Fig. 1. Árbol familiar.

\section{Caso índice (caso 1)}

JeRB. Varón de 37 años, casado y con tres hijos, uno de ellos afectado de EC (caso 5). Diagnosticado en 1974, cuando tenía 30 años de edad de enfermedad celiaca, en base a criterios clínico-analíticos y confirmados por biopsia intestinal que mostró una atrofia marcada de vellosidades intestinales (estadio 3c de Marsh). No se determinaron los DQs. Se puso a dieta sin gluten, experimentando una clara mejoría clínica, recuperando $8 \mathrm{~kg}$ de peso y desapareciendo por completo las diarreas que previamente presentaba. Estuvo asintomático hasta el año 1981, en que presentó intensos dolores abdominales, con crisis suboclusivas e importante síndrome general asociado con marcada pérdida de peso. En el estudio practicado se le detectó una tumoración a nivel de la zona proximal del intestino delgado (duodenoyeyunal), acompañada de metástasis hepáticas, cuya biopsia confirmó se trataba de un adenocarcinoma poco diferenciado, falleciendo dos meses después.

\section{Caso 2}

HRB. Varón de 70 años. Hermano. Casado y con tres hijos sanos. Tiene un nieto de cinco años hemofílico. Operado en tres ocasiones de pólipos nasales, con rinitis alérgica asociada. Diagnosticado hace 2 años de EC, por diarrea crónica de 20 años de evolución y desde entonces con DSG con buena respuesta clínica, habiendo desaparecido la diarrea y recuperado $9 \mathrm{~kg}$ de peso. No afectación cutánea asociada. Al diagnóstico presentaba una analítica general dentro de límites normales, con una TTG elevada (93,2 U/ml). Es DQ2 (+) y la biopsia duodenal mostraba una atrofia leve de las vellosidades duodenales (estadio 3A de Marsh).

\section{Caso 3}

MRB. Varón de 67 años. Hermano. Casado y con 2 hijos, uno afecto de esclerosis múltiple. Rinitis y faringitis de repetición desde la infancia. Lesiones de psoriasis generalizada, en codos, rodillas y cuero cabelludo desde hace 25 años. En el hemograma destaca la presencia de leucopenia mantenida (3.700), acompañada de ligero descenso de ferritina $(21 \mathrm{ng} / \mathrm{ml})$ sin anemia asociada y con descenso de los niveles séricos de vitamina $\mathrm{B}_{12}(177 \mathrm{pg} / \mathrm{ml})$. La TTG

Tabla I. Características clínico-analíticas del caso índice y familiares con EC

\begin{tabular}{|c|c|c|c|c|c|}
\hline Parent./caso & Edad/sexo & Clínica & $\begin{array}{c}\text { TTG } \\
(\mathrm{U} / \mathrm{ml})\end{array}$ & $D Q 2$ & $\begin{array}{c}\text { Biop. duodenal } \\
\text { (Marsh) }\end{array}$ \\
\hline Caso índice (II-8) & $37 / \mathrm{M}$ & Síndrome gral. & - & - & Adenoca. ileal \\
\hline Hermano (II-1) & $70 / \mathrm{M}$ & Diarrea & 93,2 & $(+)$ & $3 a$ \\
\hline Hermano (II-3) & $67 / \mathrm{M}$ & Psoriasis & 1,7 & $(+)$ & 0 \\
\hline Hermana (II-6) & $57 / \mathrm{M}$ & Asintomática & 1,5 & $(+)$ & 2 \\
\hline Hijo (III-10) & 29/M & PFH alteradas & 2,4 & $(-)$ & $3 b$ \\
\hline Sobrina (III-4) & $42 / F$ & Hipotiroidismo DH & 1,8 & $(-)$ & 2 \\
\hline Sobrina (III-6) & $35 / F$ & Estreñimiento & 1,9 & $(-)$ & 1 \\
\hline Sobrino (III-7) & $17 / \mathrm{M}$ & $\mathrm{DH}$ & 0,8 & $(-)$ & 0 \\
\hline Sobrino-nieto (IV-1) & 9/M & $\mathrm{DH}$ & 0,9 & $(+)$ & 1 \\
\hline
\end{tabular}

Parent: parentesco; M: masculino; F: femenino; $\mathrm{DH}$ : dermatitis herpetiforme.

La identificación de los casos afectos se relaciona con su representación en el árbol familiar. 
estaba discretamente elevada $(1,74 \mathrm{U} / \mathrm{ml})(\mathrm{N} \leq 1)$. Es DQ2 (+) y la biopsia duodenal fue interpretada como normal (estadio 0 de Marsh). Se le diagnosticó de EC hace 6 meses y se puso a DSG con buena respuesta clínica y analítica al cabo de ellos.

\section{Caso 4}

JoRB. Mujer de 57 años. Hermana. Casada y con 1 hija única de 35 años con EC (caso 6). Tuvo tres abortos (uno antes y dos después del parto de su hija). Rinitis alérgica asociada. Asintomática desde el punto de vista digestivo. En el hemograma presenta leucopenia persistente (4.450), sin anemia asociada. Presentó positividad transitoria a los anticuerpos anti-músculo liso (AML) a título medio (1/320), en una determinación realizada hace 2 años, con $\mathrm{PFH}$ normales, que posteriormente desaparecieron sin tratamiento inmunosupresor. La TTG estaba discretamente elevada $(1,5 \mathrm{U} / \mathrm{ml})$. Es DQ2 (+) y la biopsia duodenal muestra la presencia de un denso infiltrado inflamatorio crónico a nivel de la lámina propia, sin atrofia de vellosidades (estadio 2 de Marsh). Está con DSG desde hace un año, con buena respuesta.

\section{Caso 5}

CSR. Mujer de 42 años. Sobrina. Casada y con un hijo también celiaco (Caso 9). Su madre CRB de 65 años, hermana mayor del caso índice, no cumple criterios clínicoanalíticos de EC, pero presenta anticuerpos anti-tiroideos persistentemente positivos a título alto, con normofunción tiroidea. Padece rinitis crónica y elipepsia desde la infancia y presenta defectos de maduración del esmalte y lesiones cutáneas recurrentes, diagnosticadas de dermatitis herpetiforme (DH). Dolores abdominales frecuentes desde la juventud, con hábito intestinal normal. Presenta hipotiroidismo asociado en tratamiento hormonal sustitutivo con Levothroid-50 mcg (1 comp./día). Se objetivó discreta trombopenia persistente (142.000), sin leucopenia, ni anemia asociadas. La TTG ha sido ligeramente positiva $(1,8$ U/ml). Es DQ2 (-) y DQ8 (+). Las biopsias duodenales muestran la presencia de una importante inflamación crónica en la lámina propia (estadio 2 de Marsh).

\section{Caso 6}

FGR. Mujer de 35 años. Sobrina. Hija de JoRB (caso 4). Soltera. Nacida de 8 meses, con una vuelta de cordón umbilical alrededor del cuello y por parto distócico. Como consecuencia de ello, padece una parálisis cerebral con importante retraso mental y psicomotriz, camina con dificultad con muletas y utiliza parcialmente una silla de ruedas. Se encuentra muy hinchada habitualmente y le sientan muy mal las comidas. Ardores epigástricos fre- cuentes, acompañados de reflujo. Hábito intestinal muy estreñido, precisando empleo habitual de laxantes. En la analítica existe un descenso persistente de ferritina (6 ng/ml) y del IST $(13,4 \%)$ sin anemia acompañante. Presenta anticuerpos anti-tiroideos ligeramente positivos (163 U/ml), con normofunción tiroidea. La TTG es ligeramente positiva $(1,9)$, el DQ2 es negativo, siendo DQ8 (+) y las biopsias duodenales mostraron un claro aumento de los linfocitos intraepiteliales (estadio 1 de Marsh).

\section{Caso 7}

JRA. Varón de 16 años. Sobrino. Sin antecedentes de interés. Asintomático. Erupciones cutáneas generalizadas, especialmente en cara, brazos y piernas, muy pruriginosas desde la infancia, diagnosticado de dermatitis herpetiforme (DH). En la analítica destaca una ligera leucopenia (4.800), con descenso del colesterol total (116 mg/dl). La TTG fue normal $(0,88 \mathrm{U} / \mathrm{ml})$. Es DQ2 (-) y DQ8 (+). Las biopsias duodenales fueron normales (estadio 0 de Marsh). Se le puso a DSG hace 10 meses, con buena respuesta clínica y analítica, con desaparición de las lesiones cutáneas.

\section{Caso 8}

CJRM. Varón de 29 años. Hijo mediano de tres hermanos (los otros dos, están sanos). Casado y sin hijos. No bebedor, ni fumador. Amigdalitis de repetición en la infancia, siendo operado a los 6 años. Asintomático. Hábito intestinal normal. Analítica normal con ligera elevación persistente de las transaminasas. TTG positiva $(2,46)$. DQ2 (-) y DQ8 (+). La biopsia duodenal mostró atrofia moderada de las vellosidades intestinales, acompañada de infiltrado inflamatorio importante, a nivel de la lámina propia (estadio 3b de Marsh). Está con DSG desde hace 2 años, con buena respuesta clínica y analítica.

\section{Caso 9}

OVS. Varón de 9 años. Sobrino-nieto. Hijo de CRS (caso 5). Rinitis alérgica de repetición desde el año de nacer y erupciones cutáneas recurrentes, pruriginosas compatibles con DH. No molestias digestivas. Hábito intestinal normal, con ligera tendencia al estreñimiento. Analítica normal. TTG dentro de límites normales $(0,9)$. Es DQ2 (+). La biopsia duodenal fue informada como cambios mínimos (estadio 1 de Marsh). Lleva 1 año a DSG, con buena respuesta.

\section{DISCUSIÓN}

La EC es una enteropatía crónica, causada por una intolerancia permanente al gluten. El intestino delgado posee una considerable reserva funcional y ello explica que 
muchos individuos afectados presentan clínicamente pocos síntomas o están completamente asintomáticos y muchos de ellos no presentan malabsorción asociada.

La presentación clínica de la enfermedad depende de diversos factores tales como la edad de aparición, la intensidad de la sensibilidad al gluten y la cantidad de harina de cereales que se consuman. Existen algunas manifestaciones atípicas extraintestinales que son muy variadas y están posiblemente relacionadas con diferentes respuestas al gluten en diversos órganos y tejidos a distancia del intestino (13).

En los últimos años se ha puesto de manifiesto, a través de diversos estudios epidemiológicos realizados en diversas áreas geográficas, que existe un número muy elevado de casos no diagnosticados en la población general que posiblemente sea diez veces superior al número de casos diagnosticados en la actualidad (14).

Existe una elevada prevalencia acumulada en los denominados "grupos de riesgo" para la EC, entre los que se incluyen los pacientes con anemia ferropénica crónica refractarios al tratamiento sustitutivo por vía oral, osteoporosis, retraso de crecimiento, infertilidad, procesos autoinmunes asociados, e historia familiar, entre otros (15).

La diarrea ocurre en menos del $50 \%$ de los pacientes en el momento de su presentación, comparado con casi el $100 \%$ de los pacientes que eran diagnosticados en los años 60. La pérdida de peso es actualmente un hecho infrecuente y cuando se presenta, significa por lo general, que nos encontramos ante una forma de presentación grave, con una afectación intestinal extensa e importante. Por el contrario, hasta un $30 \%$ de los pacientes presentan sobrepeso al momento del diagnóstico $(16,17)$.

Por lo general, el comienzo de los síntomas es gradual y existe un intervalo de tiempo de varios años entre su aparición y el momento del diagnóstico. En ocasiones, los pacientes describen algún proceso desencadenante de sus molestias tal como un episodios de gastroenteritis aguda (GEA), un viaje al extranjero, especialmente a países en vías de desarrollo o tropicales, el estrés o cualquier procedimiento quirúrgico.

Los síntomas constitucionales tales como astenia, disminución del apetito y depresión se recogen frecuentemente, pero no son suficientes para sospechar el diagnóstico.

La presencia de dolor e hinchazón abdominal y alteración del hábito intestinal, aparecen en ausencia de malabsorción y este conjunto de síntomas es por lo general indistinguible del que presentan los pacientes con síndrome de intestino irritable (SII). Estos últimos, cumpliendo criterios de Roma II, pueden ser celiacos en un porcentaje alto, generalmente superior al $5 \%$ de los casos, por lo que deberían ser estudiados de forma rutinaria serológicamente con determinación de la TTG, para intentar descartar o confirmar dicho diagnóstico (18).

La deficiencia de hierro y ácido fólico es frecuente, bien de forma aislada o combinada, pudiendo presentarse con o sin anemia asociada. El déficit de vitamina $B_{12}$, no suele observarse porque su absorción es cofactor dependiente y se realiza a nivel del íleon terminal, que no suele estar afectado por la EC.

Se ha demostrado recientemente que la interleuquina 15 (IL-15) está muy aumentada a nivel de la mucosa intestinal de los pacientes celiacos. Dicha citoquina se expresa en las células de sistema inmune innato, tales como los entericitos y los monocitos de la lámina propia intestinal. Ello indica la importancia que juega este sistema en los estadios precoces de la patogenia de la enfermedad y sugiere la posible existencia de un mecanismo tóxico directo del gluten frente al epitelio intestinal, asociado con el daño inmunológico producido por el sistema inmune adquirido mediado por linfocitos CD-8 y otras citoquinas proinflamatorias como el TNF-alfa (19).

El punto más importante para llegar al diagnóstico de un paciente como celiaco consiste en que el médico piense en su posible existencia ante todo paciente que presente molestias digestivas frecuentes acompañadas o no de otras manifestaciones sistémicas. No existe un único procedimiento que pueda de forma definitiva diagnosticar o descartar una EC ante cada individuo en particular. Es la combinación de datos clínicos, analíticos, genéticos y anátomo-patológicos lo que nos permite por lo general llegar al diagnóstico definitivo; si bien, en casos de duda, puede ayudarnos la instauración de una dieta sin gluten (DSG), durante un tiempo mínimo de seis meses y observar su respuesta (20).

Se describen una serie de alteraciones endoscópicas duodenales en los pacientes celiacos, tales como palidez de la mucosa, "patrón en mosaico", festoneado de los pliegues, etc. Estos cambios muestran una cierta correlación con las alteraciones histológicas, pero en muchas ocasiones no aparecen y se debe biopsiar sistemáticamente el duodeno con 4-6 tomas, especialmente cuando se exploran pacientes de riesgo, para tratar de incrementar el número de diagnósticos $(21,22)$.

Los hallazgos histológicos más característicos consisten en aplanamiento o atrofia de las vellosidades, hiperplasia de las criptas e infiltración linfocitaria de la lámina propia. Existe además un aumento en el número de los linfocitos intraepiteliales (LIE), que es más evidente a nivel de la punta de las vellosidades intestinales y es de los hallazgos histológicos que aparecen más precozmente en relación con el gluten (23).

La prevalencia de la EC en familiares de primer grado ha sido analizada directamente, mediante realización de biopsia intestinal en 5 estudios amplios; 3 de ellos fueron llevados a cabo en Inglaterra en los años 70 (24-26) y los 2 restantes, se realizaron en Finlandia en la década de los 90 (27,28). El porcentaje de miembros afectos, osciló del 34 al $100 \%$ (25). El tamaño de los familiares estudiados osciló entre 29 y 182 pacientes. La prevalencia media de EC entre los familiares de primer grado, mediante su estudio a través de la biopsia intestinal, estaba comprendida entre el 5,5\% (25) hasta el 22,5\% (26), con una media acumulada del $16 \%$. 
El despistaje serológico de familiares de primer grado se ha llevado a cabo en diversos estudios que han mostrado una prevalencia media de alrededor del 12\% (29), pero cuando se incluyen también las lesiones iniciales tipo Marsh I, aumenta hasta alcanzar valores del 44,1\% (30) muy similares a los encontrados por nosotros en el presente trabajo.

La prevalencia de EC parece ser más elevada en familiares con múltiples casos conocidos (31) y algunos autores no encuentran apenas diferencias entre la prevalencia de EC en familiares de segundo grado 19,5\% (IC-95\%, $15,1-23,9)$ frente a los de primer grado que fue del $17 \%$ (IC-95\%, 6,4-27,7) (32).

También se ha observado que la edad de la población estudiada de los familiares puede influir en los resultados observados, ya que en el seguimiento de estudios de individuos que inicialmente eran negativos, posteriormente se hicieron positivos si presentaban predisposición genética adecuada (33).

La EC se asocia con el linfoma intestinal y otras formas de cánceres digestivos, especialmente adenocarcinoma de intestino delgado, de faringe y de esófago.

En una serie de 175 casos de tumores malignos del intestino delgado recogida en Inglaterra, se encontró una asociación con EC en el $13 \%$ de los casos, habiéndose diagnosticado primero la enteropatía por gluten en el $63 \%$ de los pacientes (con un intervalo de tiempo transcurrido entre el diagnóstico de ambos procesos de 8,2 años) (34).

En nuestro caso índice, el intervalo de tiempo transcurrido entre la realización de ambos diagnósticos fue muy similar, aunque algo menor (7 años).

Al igual que en esta serie, nuestro paciente tenía un adenocarcinoma de intestino delgado de localización proximal (duodeno-yeyunal), acompañado de signos clínicos de obstrucción intestinal y hemorragia digestiva. Cuando la resección quirúrgica es posible, la supervivencia es ligeramente mayor que en el caso del linfoma intestinal.

En un estudio epidemiológico recientemente publicado llevado a cabo en Suecia sobre una cohorte de 11.000 individuos celiacos, seguidos durante 31 años, encontraron un riesgo aumentado en esta población para el cáncer orofaríngeo, con una relación estandarizada de incidencia (SIR) de 2,3 y para el cáncer de esófago de 4,2. También encontraron un ligero incremento para el cáncer de colon de 1,5 , principalmente de localización proximal, pero no para el de colon descendente y recto (35).

Es por tanto obligada la realización de estudios familiares, incluyendo el mayor número posible de allegados de primero y segundo grado, ya que en casos especiales con pocos miembros puede darse la casualidad de estar todos afectos (36) porque se pueden prevenir muchas enfermedades autoinmunes y varios tipos de tumores malignos y porque la instauración de una DSG mejora notablemente la calidad de vida de los pacientes celiacos, como ha sido claramente demostrado por el Dr. Casellas y cols. recientemente (37).

\section{AGRADECIMIENTOS}

Al Prof. Joaquín Fernández Toral, Jefe de Sección de Genética Infantil del Hospital Central de Asturias, por la ayuda y colaboración prestada en la realización del árbol familiar.

\section{BIBLIOGRAFÍA}

1. Alaedini A, Green PH. Narrative review: Celiac disease: Understanding a complex autoimmune disorder. Ann Inter Med 2005; 142: 289-98.

2. Fasano A, Catasi C. Current approaches to diagnosis and treatment of celiac disease. Gastroenterology 2001; 120: 636-51.

3. Bingley PJ, Williams AJ, Norcross AJ, Unsworth DJ, Lock RJ, Ness AR, et al. Undiagnosed celiac disease at age seven: population based prospective birth cohort study. BMJ 2004; 328: 322-3.

4. Wong RC, Steele RH, Reeves GE, Wilson RJ, Pink A, Adelstein S. Antibody and genetic testing in celiac disease. Pathology 2003; 35: 285-304.

5. Green PH, Jabri B. Coeliac disease. Lancet 2003; 362: 383-91.

6. Greco L, Romino R, Coto I, Di Cosmo N, Percopo S, Maglio M, et al. The first large population based twin study of coeliac disease. Gut 2002; 50: 624-8.

7. Rodrigo L, Riestra S, Fuentes D, González S, López-Vázquez A, López-Larrea C. Diverse clinical presentations of celiac disease in the same family. Rev Esp Enferm Dig 2004; 96: 612-9.

8. Meijer JWR, Mulder CJJ, Goerres MG, Boot H, Schweizer JJ. Coeliac disease and extra-intestinal T cell lymphomas: definition, diagnosis and treatment. Scand J Gastroenterol Suppl 2004; 241: 78-84.

9. Cellier C, Delabesse E, Helmer C, patey N, Matuchansky C, Jabri B, et al. Refractory sprue, celiac disease and enteropathy associated Tcell lymphoma. Lancet 2000; 356: 260-4.

10. Fernández E, Riestra S, Rodrigo L, Blanco C, López-Vazquez A, Fuentes D, et al. Comparison of six human anti-transglutaminase ELISA-tests in the diagnosis of celiac disease in the Saharawi population. World J Gastroenterol 2005; 11: 3762-6.

11. Olerup O, Aldener A, Fogdell A. HLA-DQB1 and DQA1 typing by PCR amplification with sequence-specific primers (PCR-SSP) in 2 hours. Tissue Antigens 1993; 11: 119-34.

12. Marsh MN, Bjarnason I, Shaw J, et al. Studies of intestinal lymphoid tissue. XIV-HLA status, mucosal morphology, permeability and epithelial lymphocyte populations in first-degree relatives of patients with coeliac disease. Gut 1990; 31: 30-6.

13. James WJ, Scott BB. Coeliac disease: The cause of the various associated disorders? Eur J Gastroenterol Hepatol 2001; 13: 1119-21.

14. Catassi C, Ratsch IM, Fabiani E, Rossini M, Bordicchia F, Candela F, et al. Coeliac disease in the year 2000: Exploring the iceberg. Lancet 1994; 343: 200-3.

15. Fasano A, Berti I, Gerarduzzi T, et al. Prevalence of celiac disease in at-risk and not-at-risk groups in the United States: a large multicenter study. Arch Intern Med 2003; 163: 286-92.

16. Lo W, Sano K, Lebwohl B, Diamond B, Green PH. Changing presentation of celiac disease. Dig Dis Sci 2003; 48: 395-8.

17. Dickey W, Bodkin S. Prospective study of body mass index of patients with celiac disease. BMJ 1998; 317: 1290.

18. Sanders DS, Carter MJ, Hurlestone DP, Pearce A, Ward AM, McAlindon ME, et al. Association of adult celiac disease with irritable bowel syndrome: A case-control study in patients fulfilling Rome II criteria referred to secondary care. Lancet 2001; 358: 1504-8.

19. Maiuri L, Ciacci C, Ricciardelli I, et al. Association between innate response to gliadin and activation of pathogenetic $\mathrm{T}$ cells in coeliac disease. Lancet 2003; 362: 30-7.

20. Rodrigo L. Celiac disease in the adult (Editorial). Rev Esp Enferm Dig 2006; 98: 397-407.

21. Bardella MT, Minoli G, Radaelli F, Quatrini M, Bianchi PA, Conte D. Re-evaluation of duodenal endoscopic markers in the diagnosis of celiac disease. Gastrointest Endosc 2000; 51: 714-6.

22. Riestra S, Domínguez F, Fernández-Ruiz E, García-Riesco E, Nieto 
R, Fernández E, et al. Usefulness of duodenal biopsy during routine upper gastrointestinal endoscopy for diagnosis of celiac disease. World J Gastroenterol 2006; 12: 5028-32.

23. Jarvinen TT, Collin P, Rasmussen M, Kyronpalo S, Maki M, Partanen $\mathrm{J}$, et al. Villous tip intraepithelial lymphocytes as markers of early-stage celiac disease. Scand J Gastroenterol 2004; 39: 428-33.

24. Robinson DC, Watson AJ, Wyatt EH, Marks JM, Roberts DF. Incidence of small-intestinal mucosa abnormalities and of clinical celiac disease in the relatives of children with celiac disease. Gut 1971; 12: 789-93.

25. Rolles CJ, Myint TO, Sin WK, Anderson M. Proceedings: Family study of celiac disease. Gut 1974; 15: 827.

26. Stokes PL, Ferguson R, Holmes GK, Cooke WT. Familial aspects of celiac disease. QJM 1976; 45: 567-82.

27. Polvi A, Eland C, Koskimies S, Maki M, Partanen J. HLA DQ and DP in Finnish families with celiac disease. Eur J Immunogenet 1996; 23: 221-34

28. Holm KH. Correlation of HLA-DR alleles to jejunal mucosal morphology in healthy first-degree relatives of celiac disease patients. Eur J Gastroenterol Hepatol 1993; 5: 35-9.

29. Pittschieler K, Gentili L, Niederhofer H. Onset of coeliac disease: a prospective longitudinal study. Acta Paediatr 2003; 92: 1149-52.

30. Tursi A, Brandimarte G, Giorgetti GM, Inchingolo CD. Effectiveness of the sorbitol HSUB2 breath test in detecting histological damage among relatives of coeliacs. Scand J Gastroenterol 2003; 38: 727-31.

31. Mustalahti K, Sulkanen S, Holopainen P, Laurila K, Collin P, Partanen $\mathrm{J}$, et al. Coeliac disease among healthy members of multiple case celiac disease families. Scand J Gastroenterol 2002; 37: 161-5.

32. Book L, Zone JJ, Neuhausen SL. Prevalence of celiac disease among relatives of sib pairs with celiac disease in US families. Am J Gastroenterol 2003; 98: 377-81.

33. Farre C, Humbert P, Vilar P, et al. Serological markers and HLADQ2 haplotype among first-degree relatives of celiac patients. Dig Dis Sci 1999; 44: 2344-9.

34. Howdle PD, Jalal PK, Holmes GKT, Houlston RS. Primary smallbowel malignancy in the UK and its association with celiac disease. Q J Med 2003; 96: 345-53.

35. Askling J, Linet M, Gridley G, Halstensen TS, Ekstrom K, Ekbom A. Cancer incidence in a population-based cohort of individuals hospitalised with celiac disease or dermatitis herpetiformis. Gastroenterology 2002; 123: 1428-35.

36. Cadahía V, Rodrigo L, Fuentes D, Riestra S, De Francisco R, Fernández M. Celiac disease (CD), ulcerative colitis (UC), and primary sclerosing cholangitis (PSC) in one patient: a family study. Rev Esp Enferm Dig 2005; 97: 907-13.

37. Casellas F, López Vivancos J, Malagelada JR. Current epidemiology and accesibility to diet compliance in adult celiac disease. Rev Esp Enferm Dig 2006; 98: 408-19. 